\title{
Collisional Simulations of Cometary Nuclei
}

\author{
Sin-iti Sirono \\ Earth and Environmental Sciences, Nagoya University, Building E, \\ Nagoya 464-8602, Japan
}

\begin{abstract}
Collisional simulations of cometary nuclei (icy dust aggregates) are conducted. Conditions for sticking are determined as a function of the impact velocity and mechanical properties of nuclei. Possible collisional outcomes in three evolutionary stages of icy-dust aggregates are discussed.
\end{abstract}

Comet nuclei are porous and believed to be aggregates of icy-dust grains (Greenberg 1982). There are three stages where collisions between icy-dust aggregates are important; 1) formation of Oort cloud (collisions between cometary nuclei; $V_{\text {impact }} \sim 100 \mathrm{~ms}^{-1}$ ), 2) evolution of Kuiper belt objects (collisions between icy planetesimals; $V_{\text {impact }} \sim 10-100 \mathrm{~ms}^{-1}$ ), and 3 ) formation of icy planetesimals (collisions between icy dust aggregates; $V_{\text {impact }} \sim 10 \mathrm{~ms}^{-1}$ ). It was shown that the extent of fragmentation strongly affects the stages 1) (Stern \& Weissman 2001) and 2) (Stern \& Colwell 1997). In the stage 3), sticking of a dust aggregate is crucial for the formation of planetesimals. In this report, I investigate collisions between aggregates composed of icy dust grains and clarify conditions for sticking.

I performed collisional simulations of dust aggregates using an SPH code (Benz \& Asphaug 1994). Details of simulations will appear soon (Sirono 2004). In this code, a dust aggregate is treated as a continuum medium. This enables us to simulate aggregates of any large sizes up to $\sim 10 \mathrm{~km}$. This limit comes from ignorance of gravity force in this simulation.

There are two important mechanical properties of an aggregate to be included in the simulation. One is elasto-plastic deformation and the other is healing of fractures. An aggregate deforms elastically for sufficiently small external stresses. However, plastic deformation proceeds by re-arrangement of grains if a large stress is applied. A boundary between elastic and plastic deformation defines a yield surface, which is a hypersurface in a three dimensional space of principal components of a stress tensor. A stress tensor can be decomposed into two terms: hydrostatic pressure and deviatory stress. When pressure reaches compressive strength $\Sigma(\rho)$, irreversible compaction occurs and when pressure reaches tensile strength $-T(\rho)$, tensile fragmentation starts. For the deviatory part, we adopt the von Mises yielding criterion. If the second invariant of deviatory stress becomes larger than the square of yield strength $Y^{2}$, irreversible shear deformation takes place (Benz \& Asphaug 1994). Here I assumed $\Sigma(\rho)$ and $T(\rho)$ vary as power laws of $\rho$ as $\Sigma(\rho)=\Sigma_{0} \rho^{6}$ and $T(\rho)=T_{0} \rho^{5}$, and $Y(\rho)$ is given by $Y(\rho)=\sqrt{2 T(\rho) \Sigma(\rho) / 3}$.

When stress reaches the yield surface, fragmentation of an aggregate begins. The degree of fragmentation is expressed by the damage parameter $D$ increasing 

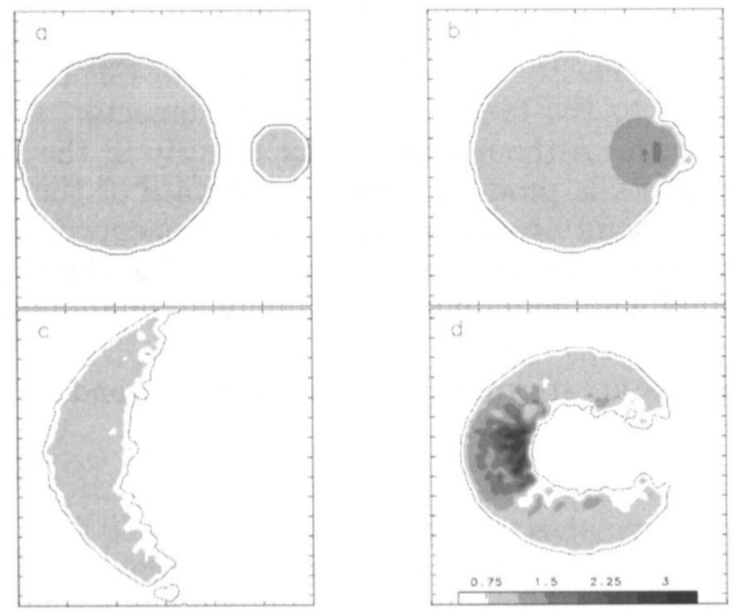

Figure 1. Density distributions of representative collisions. (a) Initial arrangement of an impactor (small sphere) and a target (large sphere). Size ratio is 10:3. (b) Sticking collision. $\Sigma_{0}=10^{-3}, T_{0}=10^{-2}$, and $M=0.13$. (c) Catastrophic distuption. $\Sigma_{0}=10^{-2}, T_{0}=10^{-3}$, and $M=0.13$. (d) Formation of a deep hole. $\Sigma_{0}=10^{-4}, T_{0}=10^{-3}$, and $M=0.4$.

from 0 to 1 as reducing the strengths and elastic moduli by a factor of $1-D$. When $D$ reaches 1 , the region cannot sustain any tensile and shear stresses.

A typical size of a composing grain is $\mu \mathrm{m}$ (Greenberg 1982). Because of its large surface to mass ratio, surface cohesion of a grain is effective. In this case, the damage due to the fracture can be restored by reconnection between grains followed by compaction of an aggregate. If this "healing effect" is effective, the damage parameter $D$ can be reduced as compaction proceeds. I conducted both cases of simulations, with and without the healing effect.

The input parameters are the initial normalized compressive strength $\Sigma_{0}$, normalized tensile strength $T_{0}$ (both quantities are normalized with bulk modulus of an aggregate), and the impact velocity $M$ which is normalized with the sound speed of an aggregate. In addition to these parameters, the impact angle is varied to simulate off-center collisions.

Figure 1 summarizes representative results of simulations. The gray contours of the figures shows density distribution of an aggregate and the scale is shown in the bottom of Fig 1d. The initial arrangement of a target (large sphere) and an impactor (small sphere) is shown in Fig 1a. A sticking case is shown in Fig 1b. The parameters are $\Sigma_{0}=10^{-3}, T_{0}=10^{-2}$, and $M=0.13$. Note that the compressive strength is larger than tensile strength in this case. On the other hand, if the tensile strength is larger than the compressive strength, the outcome is catastrophic disruption as shown in Fig 1c. In this collision, the parameters are $\Sigma_{0}=10^{-2}, T_{0}=10^{-3}$, and $M=0.13$. The healing effect is included in all three simulations shown in Fig 1 . If the healing effect is not included, it was found that the outcome is catastrophic disruption even if the strength parameters are the same to those of Fig $1 \mathrm{~b}$. 
An interesting outcome can be seen in Fig 1d. A deep hole is formed. The parameters are $\Sigma_{0}=10^{-4}, T_{0}=10^{-3}$, and $M=0.4$. The aggregate is weaker and the impact velocity is higher than those of Fig $1 \mathrm{~b}$ and c. The impactor perfectly sticks to the target. Behind the impactor, a hole is formed along the impactor path. Although we cannot identify in the figure, substantial amount of fragments is produced from the surface of the hole and floats inside the target. We might observe no apparent fragmentation like this simulation when the impactor hits the comet Tempel 1 in the Deep Impact mission (http://deepimpact.jpl.nasa.gov/) if the mechanical properties of the comet is highly compressive.

A set of conditions for sticking has been found by conducting simulations for various parameter values:

- $T_{0}>\Sigma_{0}$

- $Y_{0}>\Sigma_{0}$

- $M \lesssim 0.04$

- Healing effect.

If one of the parameters of a collision does not meet these conditions, an outcome of the collision will be cratering or catastrophic disruption. The third condition $(M \lesssim 0.04)$ comes from the results of oblique impacts. If only head-on collision is concerned, the condition is $M \lesssim 0.13$.

The important parameter is the impact velocity $M$ normalized with the sound speed of an aggregate. A possible range of bulk modulus of an icy aggregate is $8 \times 10^{6} \mathrm{erg} \mathrm{cm}^{-3}$ at packing fraction of 0.1 (=90\% porosity) to $4 \times 10^{8} \mathrm{erg} \mathrm{cm}^{-3}$ at packing fraction of 0.3 (Sirono \& Greenberg 2000). From these values, the sound speed varies from $9 \mathrm{~m} \mathrm{~s}^{-1}$ to $360 \mathrm{~m} \mathrm{~s}^{-1}$. Coming back to the three important stages of aggregate collisions and impact velocities noted in the beginning, we can draw the following conclusions.

- Formation of Oort cloud: Catastrophic disruption $(0.3<M<11)$

- Evolution of Kuiper belt objects: Catastrophic disruption $(0.03<M<$ 11)

- Formation of icy planetesimals: Sticking is possible $(0.03<M<1.1)$

\section{References}

Benz, W., \& Asphaug, E. 1994, Icarus, 107, 98

Greenberg, J. M. 1982, in Comets, ed. L. L. Wilkening, 131

Stern, S. A., \& Colwell, J. E. 1997, AJ, 114, 841

Stern, S. A., \& Weissman, P. R. 2001, Nature, 409, 589

Sirono, S. 2004, Icarus, 167, 431

Sirono, S. \& Greenberg, J. M. 2000, Icarus, 145, 230 